\title{
Mathematical and laboratory modeling of resonant impact on the spike for the purpose of grain selection
}

\author{
Arkady Soloviev ${ }^{1, *}$, Andrey Matrosov ${ }^{1}$, Ivan Panfilov ${ }^{1}$, Besarion Meskhi ${ }^{1}$, Oleg Polushkin ${ }^{1}$, \\ Dmitry Rudoy ${ }^{1}$, and Valery Chebanenko ${ }^{2}$ \\ ${ }^{1}$ Don State Technical University, 344003, Gagarin0 sq., 1, Rostov-on-Don, Russia \\ ${ }^{2}$ Southern Scientific Center of the Russian Academy of Sciences, Rostov-on-Don, Russia
}

\begin{abstract}
Mathematical and computer finite element model in the ACELAN package of resonant impact on a spike was developed and a fullscale experiment was carried out. Two installations are considered, one based on a cantilever, the free end of which acts on the spike, and the second is a semi-passive round bimorph. Excitation of vibrations is carried out using an actuator based on piezoceramic elements. In the first installation, low-frequency vibrations of the stem with a spike are excited and the resonance frequency is determined at which only an spike with grain performs intense vibrations. The second installation is designed to excite high-frequency vibrations at which resonant movements of the grains themselves arise. The purpose of both installations is to separate the grain from the spike using resonance phenomena.
\end{abstract}

\section{Introduction}

The urgent task of today in the grain sector of agriculture is the task of ensuring lowenergy and low-traumatic forced extraction of grain from the spike. This requires not only the improvement of existing methods and methods of grain harvesting [1 - 7], but also the development of new highly effective physical and mechanical methods of its harvesting and, accordingly, the design of appropriate equipment [8-12].

The solution of this problem, on the one hand, requires the development and creation of adequate analytical and numerical physical and mechanical models and models based on CAD-CAE complexes capable of describing the interactions between the elements of the stem-spike-grain system, and at the same time, on the other hand physical and mechanical models of the process of impact on the stem-spike-grain system of the working bodies of grain harvesting equipment. The first stage of the work is the theoretical and experimental confirmation of the resonance modes of action on the spikes with grain. For this purpose, two laboratory installations have been developed, in which the power elements are piezo actuators, a mathematical and computer model has been developed in the ACELAN package of resonant action on a spike and a field experiment has been carried out.

\footnotetext{
* Corresponding author: solovievarc@gmail.com
} 


\section{Formulation of the problem}

The model of the effect of a piezo actuator on a fragment of a grain mass is built within the framework of the mechanics of a deformable solid. A rigorous mathematical model of the dynamics of a spike and a piezo actuator can be constructed within the framework of a continuous medium and, in particular, the linear theory of elasticity and electroelasticity. Such a model is a boundary-value problem or an initial-boundary value problem and for complex geometry, its solution can only be constructed numerically. In this work, the finite element method and its implementation in the ACELAN package are used as a numerical solution method. Continuous and discrete models used in the ACELAN package are given below.

\section{Continuous problem setting}

In this section, linear equations of the theory of elasticity and electroelasticity are used taking into account the dissipation of energy adopted in the ACELAN and ANSYS packages [13], as well as the equations of motion of liquid and gaseous media in the acoustic approximation [14].

For piezoelectric media:

$$
\begin{gathered}
\rho \ddot{u}_{i}+\alpha \rho \dot{u}_{i}-\sigma_{i j, j}=f_{i} ; D_{i, i}=0 \\
\sigma_{i j}=c_{i j k l}\left(\varepsilon_{k l}+\beta \dot{\varepsilon}_{k l}\right)-e_{i j k} E_{k} ; D_{i}+\varsigma_{d} \dot{D}_{i}=e_{i k l}\left(\varepsilon_{k l}+\varsigma_{d} \dot{\varepsilon}_{k l}\right)+\ni_{i k} E_{k}, \\
\varepsilon_{k l}=\left(u_{k, l}+u_{l, k}\right) / 2 ; \\
E_{k}=-\varphi_{, k},
\end{gathered}
$$

where $\rho$ - material density; $u_{i}$ - displacement vector components; $\sigma_{i j}-$ stress tensor components; $f_{i}$ - components of the density vector of mass forces; $D_{i}-$ electric induction vector components; $c_{i j k l}$ - components of the fourth rank tensor of elastic modules; $e_{i j k}-$ components of the tensor of the third-rank piezomodules; $\varepsilon_{i j}-$ strain tensor components; $E_{i}$ - components of the electric field strength vector; $\varphi$ - electrical potential; $\ni_{i j}$ - components of the second-rank tensor of dielectric constants; $\alpha, \beta, \varsigma_{d}-$ non-negative damping factors (at ANSYS $\varsigma_{d}=0$ ).

For elastic medium:

$$
\begin{aligned}
& \rho \ddot{u}_{i}+\alpha \rho \dot{u}_{i}-\sigma_{i j, j}=f_{i} ; \\
& \sigma_{i j}=c_{i j k l}\left(\varepsilon_{k l}+\beta \dot{\varepsilon}_{k l}\right) ; \\
& \varepsilon_{k l}=\left(u_{k, l}+u_{l, k}\right) / 2 ;
\end{aligned}
$$

In the static case, there are no terms with the time derivative, in the modal analysis, in 
addition, there are no volume forces, and in the harmonic analysis, the replacement

$$
\dot{a} \rightarrow i \omega a \text { and } \ddot{a} \rightarrow-\omega^{2} a
$$

Let us consider in more detail the mechanical and electrical boundary conditions. When defining mechanical boundary conditions, the body boundary is represented as a union of non-intersecting regions $S=S_{u} \cup S_{t} \cup S_{u t}$, on which the following conditions are set:

- condition for fixing the border or specified displacements

$$
\left.u_{i}\right|_{S_{u}}=u_{i}^{0}
$$

- condition of force action, at which the components of the vector of mechanical stresses are given

$$
t_{i}=\left.\sigma_{i j} n_{j}\right|_{S_{t}}=p_{i}
$$

- condition of smooth contact with an absolutely rigid body is the equality to zero of normal displacements and tangential stresses

$$
u_{n}=\left.u_{i} n_{i}\right|_{S_{u t}}=0, \quad t_{\tau}^{(k)}=\left.\sigma_{i j} n_{j} \tau_{i}^{(k)}\right|_{S_{u t}}=0
$$

Condition (1.9) is also posed on the axis of symmetry when solving the axisymmetric problem. When solving the problem of modal analysis, all boundary conditions are homogeneous (equal to zero). The conditions of joining (continuity of certain quantities) during the transition to the FE model are fulfilled automatically, so we will not dwell on them in detail.

$$
\text { Electrical boundary conditions are specified on a set of regions } S=\bigcup_{m=1}^{M} S_{E_{m}} \bigcup S_{D}
$$
, on which the following conditions are set:

- condition on metallized surfaces (electrodes) when a known electric potential is given

$$
\left.\varphi\right|_{S_{E_{m}}}=\varphi_{m}^{0}=\mathrm{const}
$$

-condition on non-electroded sections of the boundary when, with a certain degree of accuracy, the normal component of the electric induction vector can be considered equal to zero

$$
\left.D_{n}\right|_{S_{D}}=\left.D_{i} n_{i}\right|_{S_{D}}=D_{n}^{e} \quad(=0)
$$

The homogeneous condition (1.11) is also posed on the axis of symmetry when solving the axisymmetric problem.

- condition for determining the unknown electric potential, which is formulated in addition to condition (1.10) on an electrode connected to an external electric circuit, in particular, if the electrode is free, then the charge or current is zero $(=0)$ 


$$
\int_{E_{m}} \dot{D}_{n} d S=I_{m} \quad(=0)
$$

where $I_{m}$ - current in the circuit, which in the case of a free electrode is zero.

Finite element approximation in ACELAN

To solve dynamic problems of acoustoelectroelasticity, we will use the FEM in the classical Lagrangian formulation. A fairly complete and consistent presentation of the FEM for piezoelectrics and its implementation in the ACELAN package is presented in [13], [15].

Below, according to [13], the FE formulation of the problems considered in the work is given. When passing from the continual formulation to the FE, a coordinated discretization of geometric regions is carried out - partition into finite elements (triangulation) with a certain set of geometric points - nodes, as a result of which the boundary value problem (1.1) - (1.12) using the representation

$$
\mathbf{u}(\mathbf{x}, t)=\mathbf{N}_{u}^{T}(\mathbf{x}) \cdot \mathbf{U}(t) ; \varphi(\mathbf{x}, t)=\mathbf{N}_{\varphi}^{T} \cdot \mathbf{\Phi}(t) ; \psi(\mathbf{x}, t)=\mathbf{N}_{\psi}^{T}(\mathbf{x}) \cdot \mathbf{\Psi}(t),
$$

where $\mathbf{x}$-spatial coordinates; $t$ - time; $\mathbf{N}_{u}^{T}$ - shape function matrix for displacement field $\mathbf{u}=\left(u_{1}, u_{2}, u_{3}\right) ; \mathbf{N}_{\varphi}^{T}, \mathbf{N}_{\psi}^{T}$ - row vector of the shape functions for the fields of the electric potential $\varphi$ and the velocity potential in the acoustic medium $\psi, \boldsymbol{U}(t), \Phi(t)$, $\boldsymbol{\Psi}(t)$ - global vectors of the corresponding nodal degrees of freedom.

It is essential that approximations (1.13) are specified in those regions where there are corresponding physical fields, and may differ for different finite elements. An approximation of the FEM (1.13) of generalized statements of dynamic problems (1.1) (1.12), including the main principal and natural boundary conditions, is reduced to a system of ordinary differential equations with respect to the nodal unknowns

$$
\mathbf{a}=\lfloor\mathbf{U}, \boldsymbol{\Phi}, \boldsymbol{\Psi}\rfloor^{T}
$$

$$
\mathbf{M} \cdot \ddot{\mathbf{a}}+\mathbf{C} \cdot \dot{\mathbf{a}}+\mathbf{K} \cdot \mathbf{a}=\mathbf{F},
$$

where global matrices $\mathbf{M}, \mathbf{C}, \mathbf{K}$, which, by analogy with the FEM for elastic media, can be called matrices of mass, damping, stiffness, and the column of the right-hand sides have a block structure of the form:

$$
\begin{aligned}
\mathbf{M} & =\left(\begin{array}{ccc}
\mathbf{M}_{u u} & 0 & \tilde{\mathbf{R}}_{u \psi} \\
0 & 0 & 0 \\
\tilde{\mathbf{R}}_{u \psi}^{T} & 0 & -\mathbf{M}_{\psi \psi}
\end{array}\right), \quad \mathbf{C}=\left(\begin{array}{ccc}
\mathbf{C}_{u u} & 0 & \mathbf{R}_{u \psi} \\
\boldsymbol{S}_{\mathrm{d}} \mathbf{K}_{u \varphi}^{T} & 0 & 0 \\
\mathbf{R}_{u \psi}^{T} & 0 & -\mathbf{C}_{\psi \psi}
\end{array}\right), \\
\mathbf{K} & =\left(\begin{array}{ccc}
\mathbf{K}_{u u} & \mathbf{K}_{u \varphi} & 0 \\
\mathbf{K}_{u \varphi}^{T} & -\mathbf{K}_{\varphi \varphi} & 0 \\
0 & 0 & -\mathbf{K}_{\psi \psi}
\end{array}\right), \quad \mathbf{F}=\left\{\begin{array}{c}
\mathbf{F}_{u} \\
\mathbf{F}_{\varphi}+\boldsymbol{\varsigma}_{\mathrm{d}} \dot{\mathbf{F}}_{\varphi} \\
0
\end{array}\right),
\end{aligned}
$$


where the FE of the mass matrix $\mathbf{M}_{\mathcal{u} u}$, damping $\mathbf{C}_{\mathcal{u} u}$ and hardness $\mathbf{K}_{\boldsymbol{u} u}$ in (1.15) are the same as in structural analysis. These matrices are symmetric and non-negative definite, and $\mathbf{M}_{u u}>0$. Matrixes $\mathbf{K}_{u \varphi}$ and $\mathbf{K}_{\varphi \varphi}$ are determined by the piezoelectric effect and reflect the piezoelectric and dielectric properties, respectively, moreover $\mathbf{K}_{\varphi \varphi}$ is symmetric and non-negative definite: $\mathbf{K}_{\varphi \varphi} \geq 0$. (Here are the inequalities $\mathbf{B}>0$ or $\mathbf{B} \geq 0$ mean that the matrix $\mathbf{B}$ respectively positive definite or semidefinite). Vectors $\mathbf{F}_{\mathcal{u}}$ and $\mathbf{F}_{\varphi}$ are formed as a result of taking into account mechanical and electrical influences. Matrixes $\mathbf{M}_{\psi \psi}, \mathbf{K}_{\psi \psi}, \tilde{\mathbf{R}}_{u \psi}, \mathbf{R}_{u \psi}, \mathbf{C}_{\psi \psi}$ conditioned by acoustic environment. Matrixes $\mathbf{M}, \mathbf{C}$ and $\mathbf{K}$ are obtained as a result of the process of ensemble local matrices of all finite elements.

As can be seen from (1.15) in the FE packages ANSYS and ACELAN, the simplest models of attenuation are adopted. At the same time, in ANSYS, in piezoelectric, damping is taken into account only through elastic properties - submatrix $\mathbf{C}_{u u}$, which, in accordance with the method of accounting for Rayleigh damping, can be represented as:

$$
\mathbf{C}_{u u}=\sum_{j}\left(\alpha_{j} \mathbf{M}_{u u j}+\beta_{j} \mathbf{K}_{u u j}\right)
$$

where $\alpha_{j}$ and $\beta_{j}$-damping factors; $\mathbf{M}_{u u j}$ and $\mathbf{K}_{u u j}$ structural FE matrices of mass and stiffness $j$-th submatrices, respectively, and the remaining submatrices in (1.15) are described in [16].

When considering ideal materials, the resonance region is inaccessible for investigation, since the amplitudes of various quantities tend to infinity. However, for using structures practice, the numerical values of these quantities are extremely important. For example, the amplitudes of mechanical stresses that determine the strength of structures at resonance. Energy losses of various kinds in real material lead to the final values of these quantities. In the region of low and medium frequencies, mechanical energy losses make the greatest contribution in solids. For a quantitative description of this type of energy loss, the concept of the mechanical quality factor of the material is introduced $Q_{M}$. Determine the width of the resonance curve $\Delta \omega$ as the difference in frequencies corresponding to the half power level at the resonance frequency $\omega_{0}$. Then the quality factor is defined as

$$
\Delta \omega=\frac{\omega}{Q_{M}}
$$

In FE packages ANSYS and ACELAN, the attenuation coefficients are introduced to describe the loss of mechanical energy $\alpha, \beta$, which, assuming the same quality factor at the first two resonant frequencies $f_{r 1}, f_{r 2}$ expressed in terms of the quality factor as follows: 


$$
\alpha=\frac{2 \pi f_{r 1} f_{r 2}}{Q\left(f_{r 1}+f_{r 2}\right)}, \quad \beta=\frac{1}{2 \pi Q\left(f_{r 1}+f_{r 2}\right)}
$$

Characteristic feature of the form of equations (1.18), (1.19) in ACELAN and ANSYS is the symmetry of the matrices $\mathbf{M}$ and $\mathbf{K}$ and their similar saddle structure. The latter means that the matrixes $\mathbf{M}$ and $\mathbf{K}$ after some simultaneous rearrangement of their rows and columns take the following block forms:

$$
\mathbf{A}=\left(\begin{array}{cc}
\mathbf{B} & \mathbf{H} \\
\mathbf{H}^{T} & -\mathbf{S}
\end{array}\right) ; \quad \mathbf{B}=\mathbf{B}^{T} ; \mathbf{S}=\mathbf{S}^{T} \quad \mathbf{B} \geq 0 ; \mathbf{S} \geq 0
$$

and the sizes of all blocks for matrices $\mathbf{M}, \mathbf{K}$, and also for the matrix $\mathbf{C}$, are the same.

For steady-state oscillations, the equations are obtained by replacing

$$
\mathbf{F}_{u}=\widetilde{\mathbf{F}}_{u}(\mathbf{x}) \exp (j \omega t) ; \mathbf{F}_{\phi}=\tilde{\mathbf{F}}_{\phi}(\mathbf{x}) \exp (j \omega t) ; \mathbf{a}=\tilde{\mathbf{a}}(\mathbf{x}) \exp (j \omega t)
$$

are described by the following system of linear algebraic equations (SLAE)

$$
\begin{gathered}
\mathbf{K}_{c} \cdot \widetilde{\mathbf{a}}=\widetilde{\mathbf{F}}_{c} ; \widetilde{\mathbf{F}}_{c}=\left\lfloor\widetilde{\mathbf{F}}_{u}, \widetilde{\mathbf{F}}_{\varphi}, 0\right]^{T} \\
\mathbf{K}_{c}=\left(\begin{array}{ccc}
\mathbf{K}_{u u c} & \mathbf{K}_{u \varphi} & \mathbf{K}_{u \psi c} \\
\mathbf{K}_{u \varphi}^{T} & -\mathbf{K}_{\varphi \varphi c} & 0 \\
\mathbf{K}_{u \psi c}^{T} & 0 & -\mathbf{K}_{\psi \psi c}
\end{array}\right) \\
\mathbf{K}_{\eta \eta c}=-\omega^{2} \mathbf{M}_{\eta \eta}+i \omega \mathbf{C}_{\eta \eta}+\mathbf{K}_{\eta \eta} ; \\
\mathbf{K}_{u \psi c}=-\omega^{2} \widetilde{\mathbf{R}}_{u \psi}+i \omega \mathbf{R}_{u \psi} ; \mathbf{K}_{\varphi \varphi c}=\frac{1}{\left(1+i \omega \zeta_{d}\right)} \mathbf{K}_{\varphi \varphi}
\end{gathered}
$$

If we use real arithmetic, then problem (1.21) is a SLAE with respect to the vector of real and imaginary parts of the nodal degrees of freedom a with a matrix that does not have positive definiteness and does not have a saddle structure.

In conclusion of this subsection, let us note some features of modal analysis in ANSYS and ACELAN. This analysis is designed to find natural or resonant frequencies of elastic bodies or frequencies of electrical resonances and antiresonances for piezoelectric bodies. Electrical resonance frequencies $f_{r i}=\omega_{r i} /(2 \pi)$ and antiresonances $f_{a i}=\omega_{a i} /(2 \pi)$ are the most important characteristics of piezoelectric devices, and are also important when performing non-stationary analysis, for example, to determine the damping factors. These frequencies are the natural frequencies of piezoelectric devices and are calculated without taking into account the effects of damping and interaction with acoustic media. Thus, these frequencies can be determined from the FEM as a result of performing modal analysis from the solution of the generalized eigenvalue problems obtained from (1.25), (1.26) of the form (without taking into account acoustic media) 


$$
\begin{gathered}
\mathbf{K} \cdot \mathbf{a}=\omega^{\mathbf{2}} \mathbf{M} \cdot \mathbf{a} \\
\mathbf{M}=\left(\begin{array}{cc}
\mathbf{M}_{u u} & 0 \\
0 & 0
\end{array}\right), \quad \mathbf{K}=\left(\begin{array}{cc}
\mathbf{K}_{u u} & \mathbf{K}_{u \varphi} \\
\mathbf{K}_{u \varphi}^{*} & -\mathbf{K}_{\varphi \varphi}
\end{array}\right), \quad \mathbf{a}=\left\{\begin{array}{l}
\mathbf{U} \\
\Phi
\end{array}\right\}
\end{gathered}
$$

and the matrices $\mathbf{K}_{u \varphi}$ and $\mathbf{K}_{\varphi \varphi}$ when finding the frequencies of electrical resonances and when finding the frequencies of antiresonances differ.

As a result of the modal analysis, the spectrum of natural frequencies is found $\omega_{k}$ and the corresponding natural vibration modes.

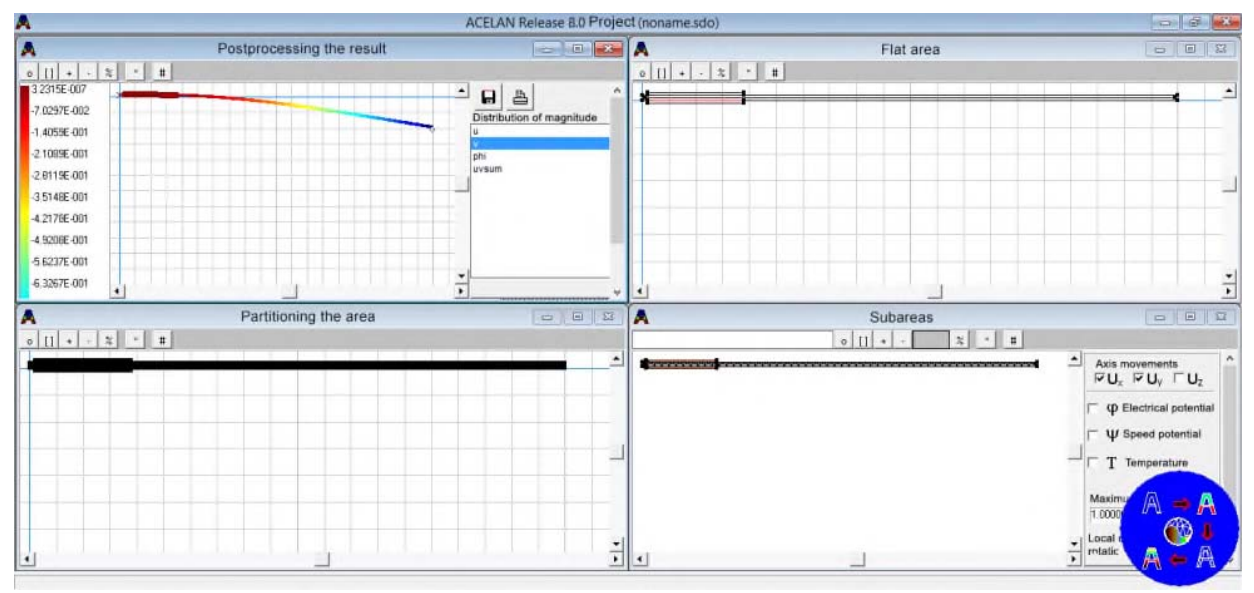

Fig. 1. ACELAN package interface (cantilever oscillation)

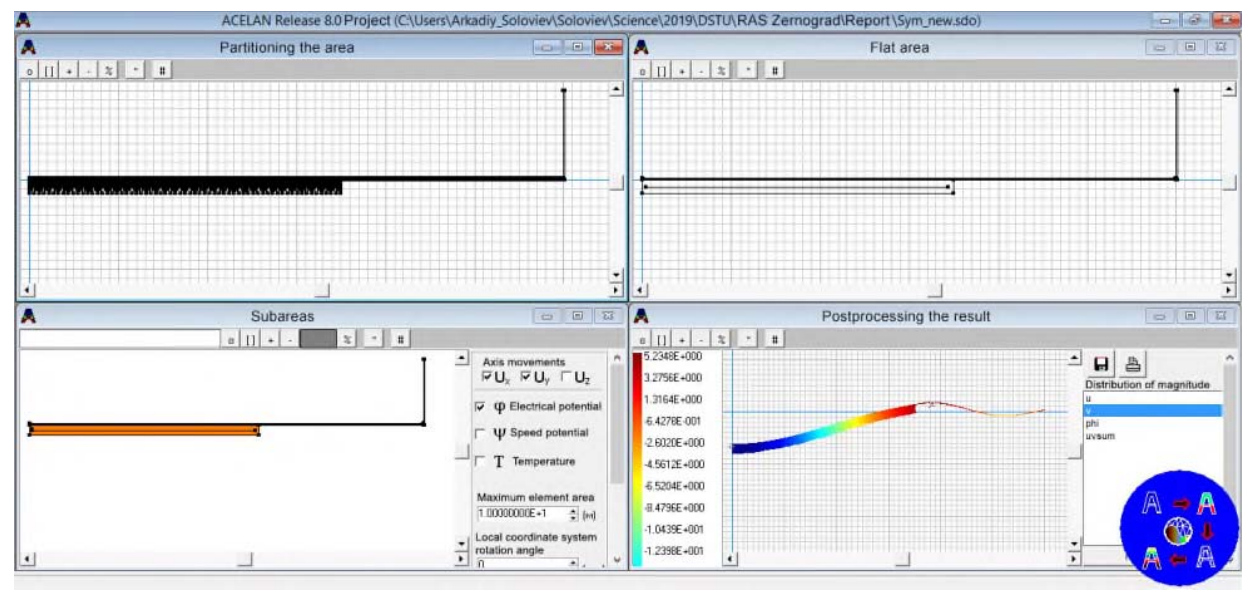

Fig. 2. ACELAN package interface (bimorph oscillation)

Figures 1 and 2 show the graphical interface of the ACELAN package when solving two tasks of modal analysis: oscillations of a cantilever with piezoelements and oscillations of a circular bimorph, respectively, these tasks are discussed in more detail below in Chapter 2. 
2. Calculation in CAE of a complex of resonances of the piezoactuator-spike system and refinement of the parameters of the resonance effect on a spike based on a natural experiment

\subsection{Calculation of a system based on a piezoactive cantilever}

The first piezo actuator is a cantilever-mounted plate with piezoelements glued at the point of attachment, to the electrodes of which an alternating voltage is applied (Fig. 2.1), which excites its bending vibrations.

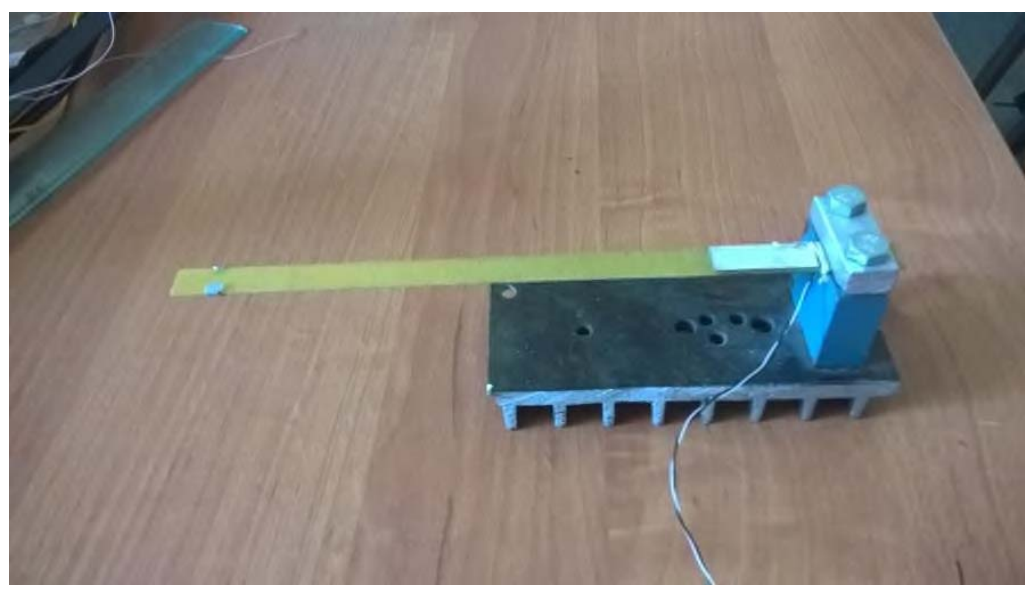

Fig. 2.1. Cantilever-based piezo actuator

The free end of the piezo actuator acts on the ear and excites its bending vibrations. The calculation of the vibration frequency of the piezo actuator was carried out in the ACELAN package.

Figures 2.2 and 2.3 show a diagram of the piezo actuator geometry and a finite element mesh, respectively. The frequency of the device changes due to the use of different designs (the length of the middle passive layer, the size of the piezoelectric elements) and the substrate material (fiberglass, duralumin), as well as by attaching an inertial mass.

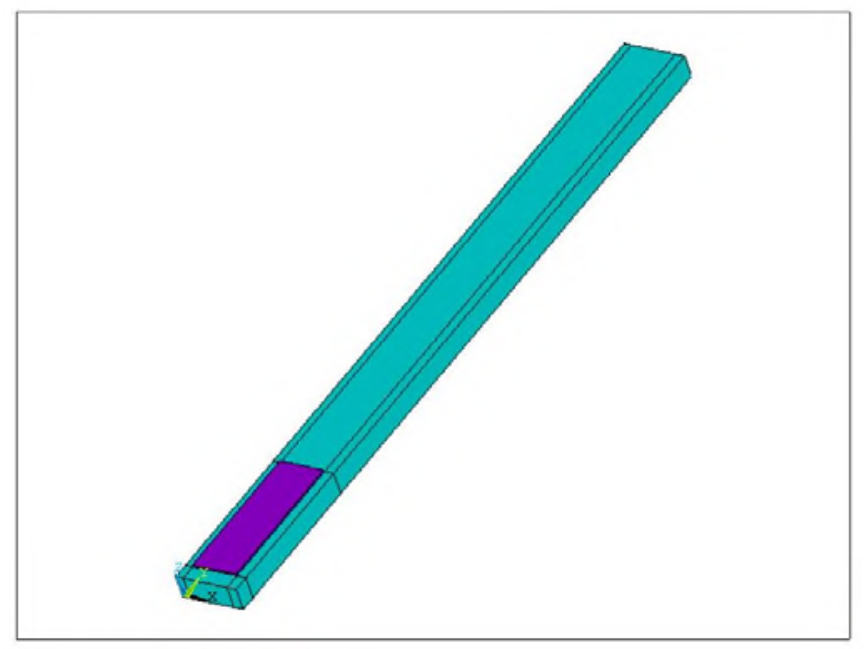

Fig. 2.2. Cantilever-based piezo actuator geometry 


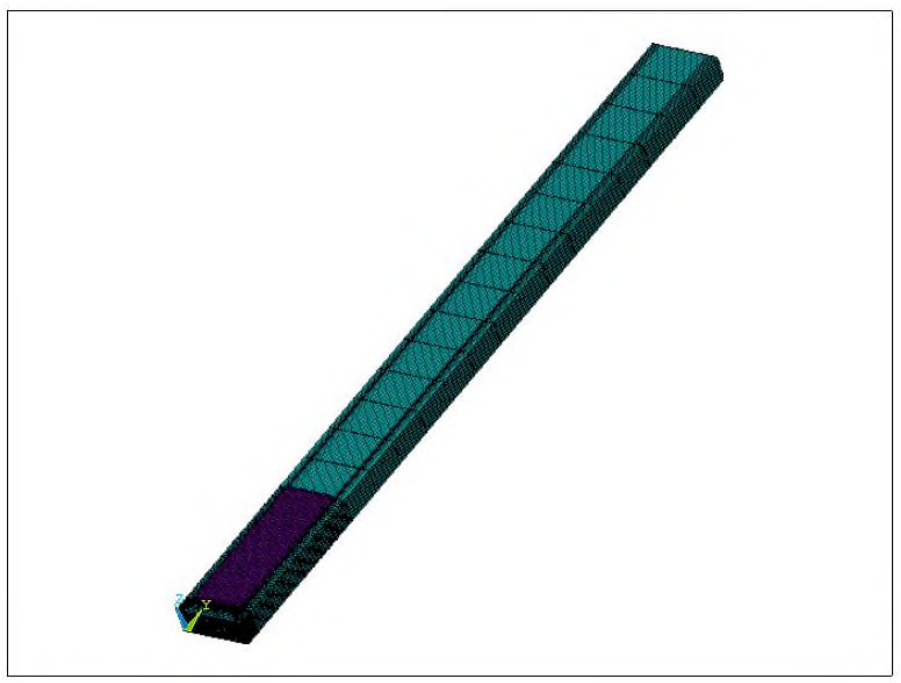

Fig. 2.3. Finite element grid of piezo actuator

Table 1 shows the first three resonant frequencies and vibration modes of a piezo actuator with a glass fiber laminate substrate PZT-4 piezoceramic for the following

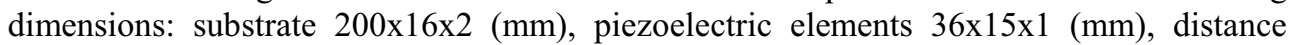
from the embedment $2 \mathrm{~mm}$.

Table 1. Natural frequencies and vibration modes

\begin{tabular}{|l|l|l|l|}
\hline № & $\begin{array}{l}\text { Resonan } \\
\text { ce } \\
\text { frequenc } \\
\mathbf{y , ~ H z}\end{array}$ & Natural mode of vibration (distribution of vertical displacements) \\
\hline 1 & 34.76 & & \\
\hline 2 & 200.1 & & \\
\hline 3 & 458.4 & & \\
\hline
\end{tabular}


Table 2. Frequency dependence on the length of the plate (fiberglass)

\begin{tabular}{|c|c|c|c|}
\hline $\begin{array}{c}\text { Substrate length } \\
(\mathbf{m m})\end{array}$ & $\mathbf{1}$ & $\mathbf{2}$ & $\mathbf{3}$ \\
\hline 100 & 189.9 & 579.2 & 1735.9 \\
\hline 200 & 34.76 & 200.1 & 458.4 \\
\hline 400 & 7.26 & 44.91 & 123.8 \\
\hline
\end{tabular}

Table 2 shows the dependences of natural frequencies on the length of the substrate for the same material, and in Table 3 for a substrate made of duralumin

Table 3. Frequency dependence on the length of the plate (duralumin)

\begin{tabular}{|c|c|c|c|}
\hline $\begin{array}{c}\text { Substrate length } \\
(\mathbf{m m})\end{array}$ & $\mathbf{1}$ & $\mathbf{2}$ & $\mathbf{3}$ \\
\hline 100 & 285.6 & 1018.2 & 2882.4 \\
\hline 200 & 560.8 & 319.4 & 767.3 \\
\hline 400 & 12.02 & 74.1 & 203.68 \\
\hline
\end{tabular}

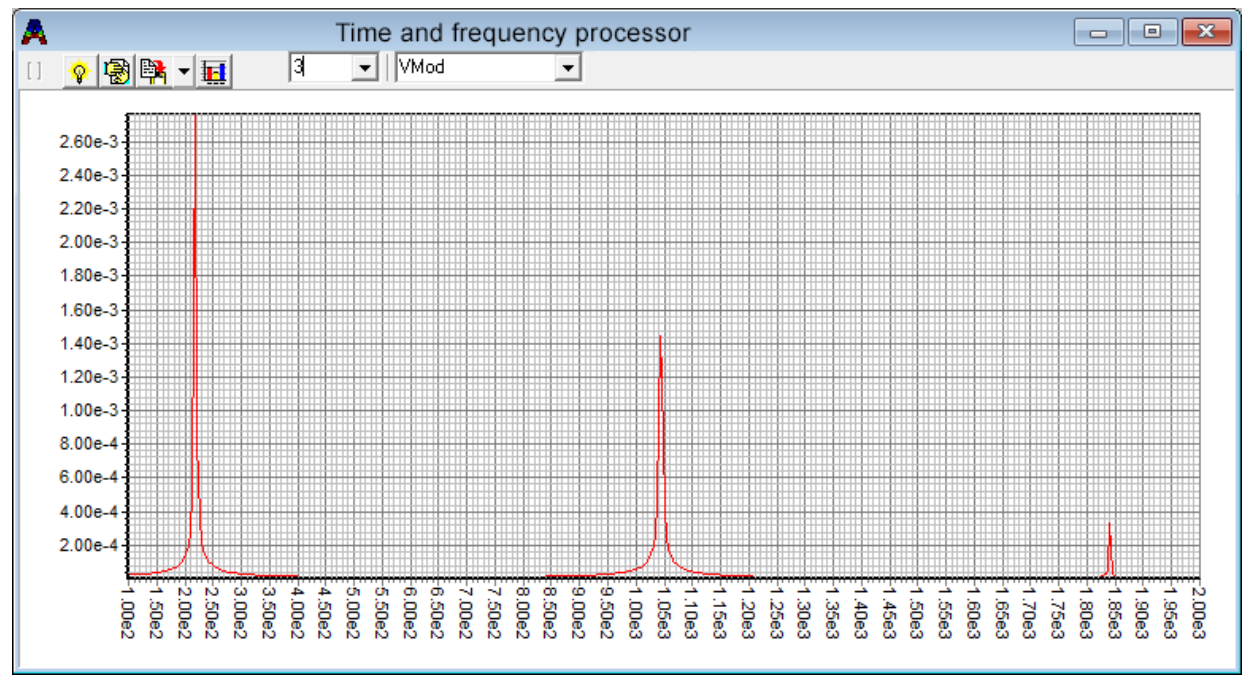

Fig. 2.4. AFC of the vertical displacement of the plate end

Figure 2.4 shows the frequency response of the vertical displacement of the free end of the piezo actuator, which acts on the ear, on the electrodes of which an electric voltage difference of $100 \mathrm{~V}$ is set.

\subsection{System calculation based on a round bimorph}

The second laboratory setup for resonant mechanical action on fragments of the grain mass is a cylindrical container with a metal bottom, to which a round disc-shaped piezoelement is glued (Figure 2.5) 


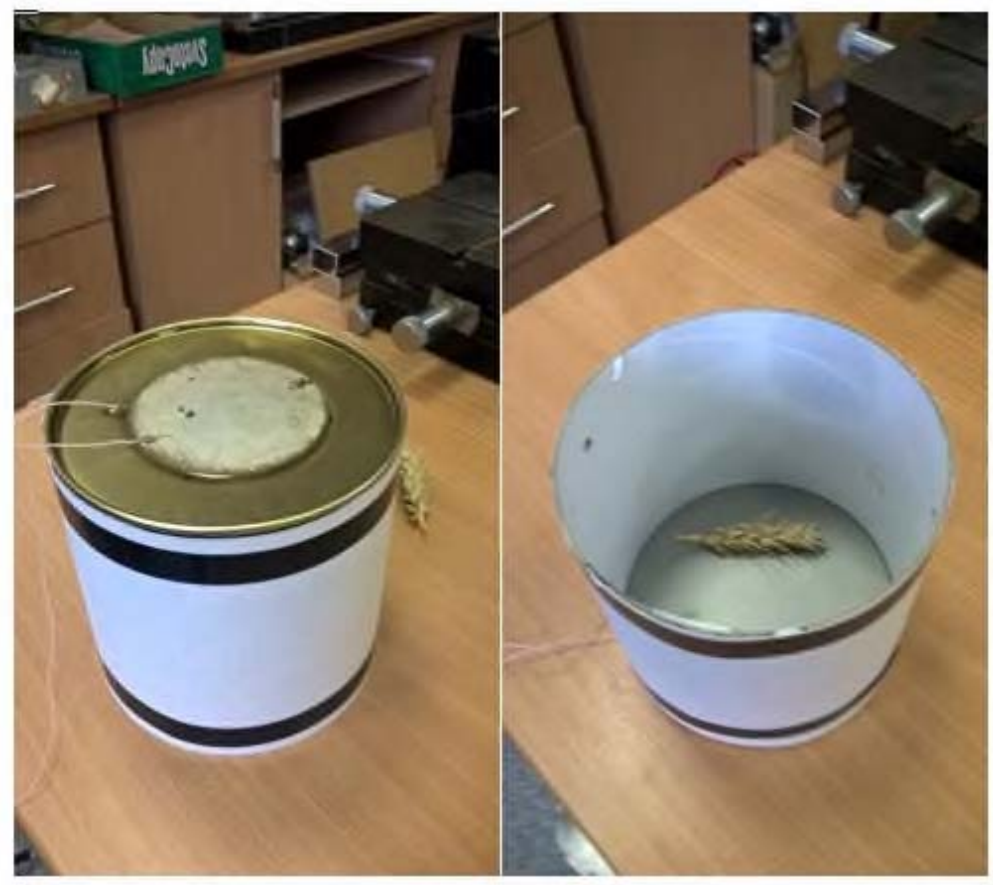

Fig. 2.5. Piezo actuator based on a round bimorph

The dynamic characteristics of this structure are calculated in the ACELAN package in an axisymmetric setting. Figure 2.6 shows a half of the axial section of a bimorph, on which the piezoelectric element is marked in light brown.

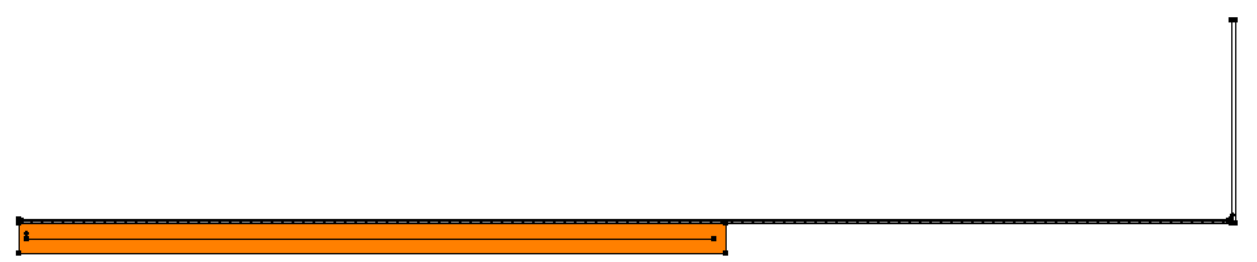

Fig. 2.6. Half axial section of a round bimorph

Figure 2.7 shows the finite element mesh of the model in the ACELAN package.

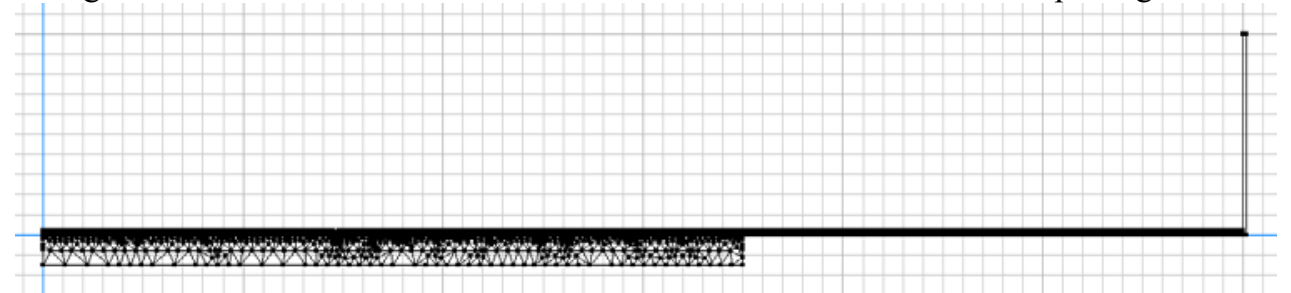

Fig. 2.7. Course element mesh for calculating a round bimorph

Figures 2.8-2.10 show the waveforms of telecommunication modes, at frequencies of $2.774 \mathrm{kHz}, 11.388 \mathrm{kHz}$ and $23.176 \mathrm{kHz}$, respectively.. 


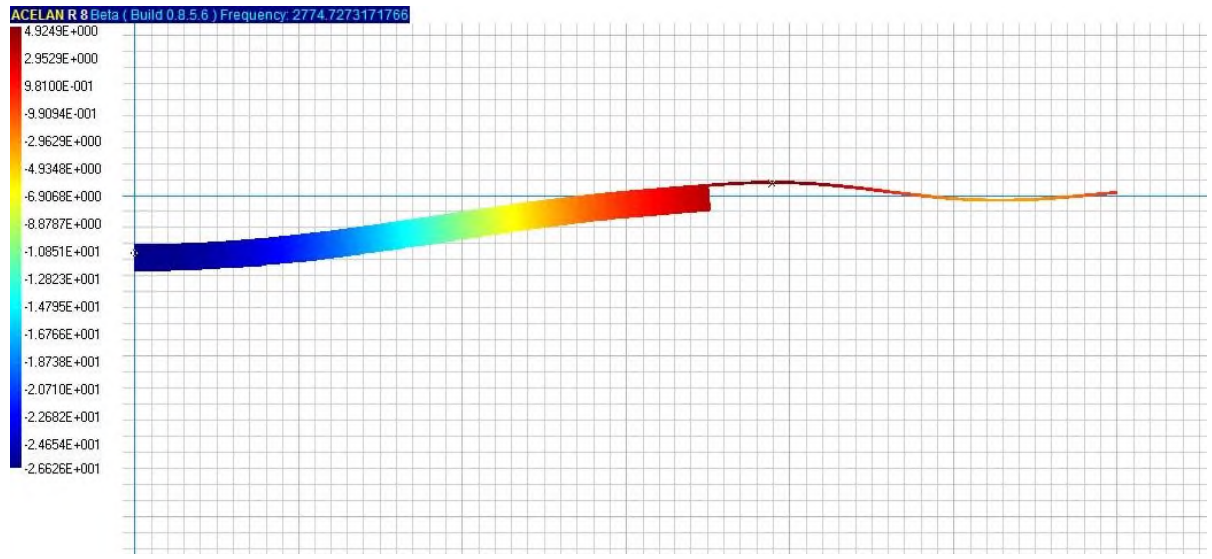

Fig. 2.8. The first electrically connected round bimorph mode

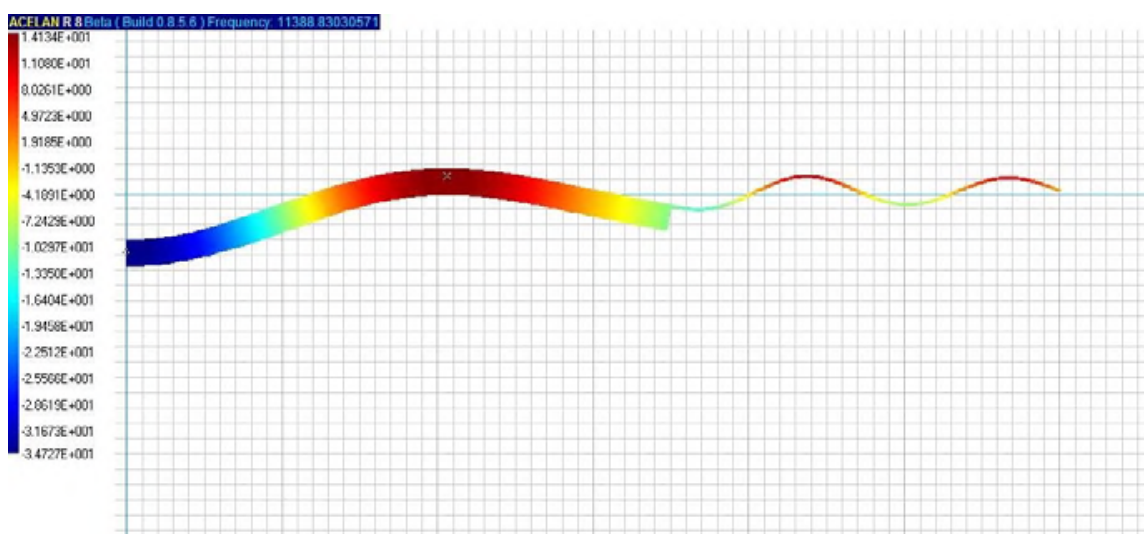

Fig. 2.9. The second electrically connected round bimorph mode

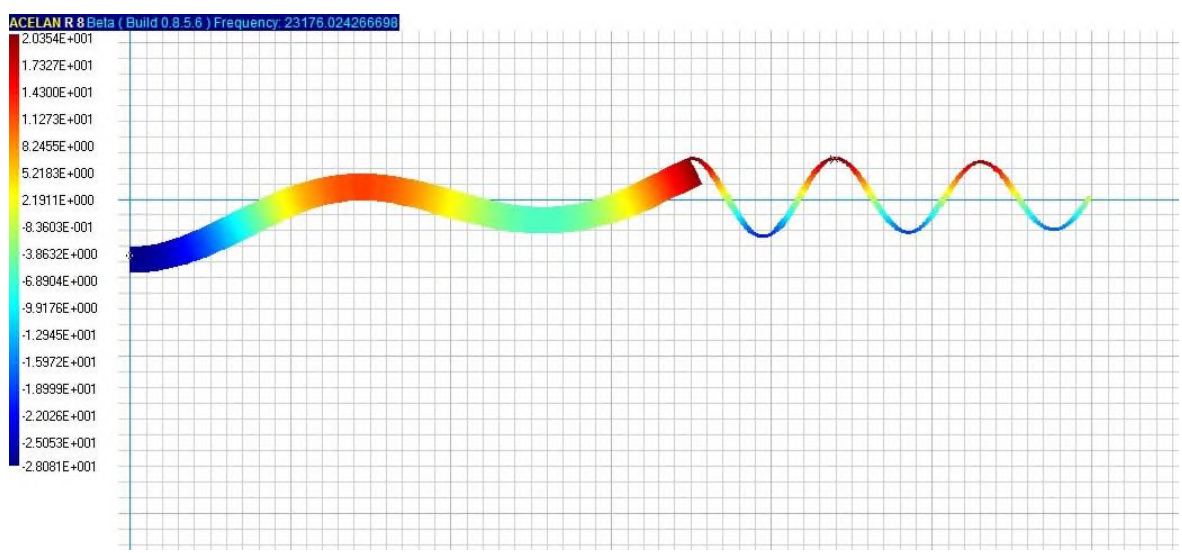

Fig. 2.10. The third electrically connected round bimorph mode 


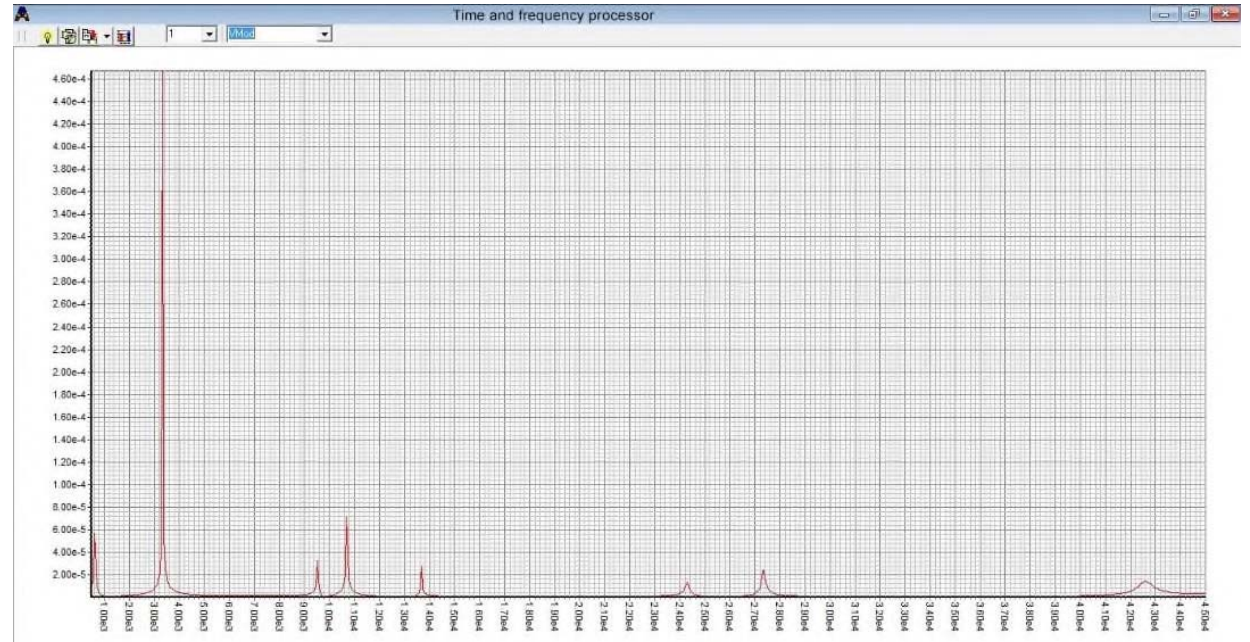

Fig. 2.11. Frequency response of axial displacement of a round bimorph

Figure 2.11 shows the frequency response of the vertical displacement of the central point of the bimorph under harmonic excitation by a potential difference across the electrodes of a $100 \mathrm{~V}$ piezoelectric element.

\subsection{Experimenting}

Full-scale experiments were carried out on the installations shown in Figures 2.1 and 2.5. Figure 2.12 shows a diagram of the test bench.

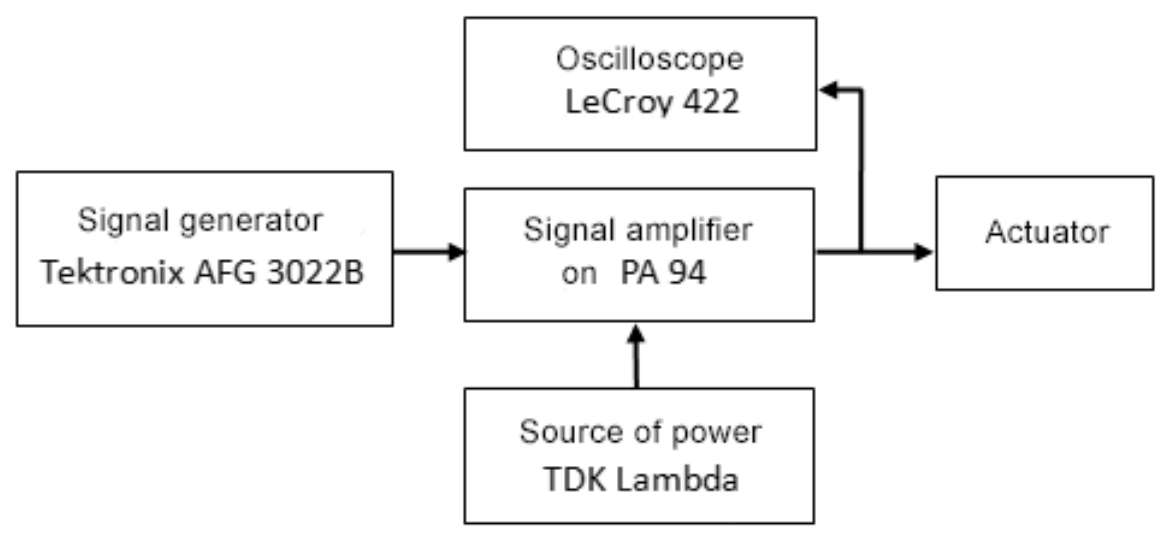

Fig. 2.12. Test bench layout

Electronic equipment for excitation of mechanical vibrations consists of a Tektronix AFG 3022B signal generator, a signal amplifier based on PA 94 piezo drivers (Apex Co., USA) and TDK Lambda power supplies. The control signal is monitored using a LeCroy 422 digital oscilloscope. 


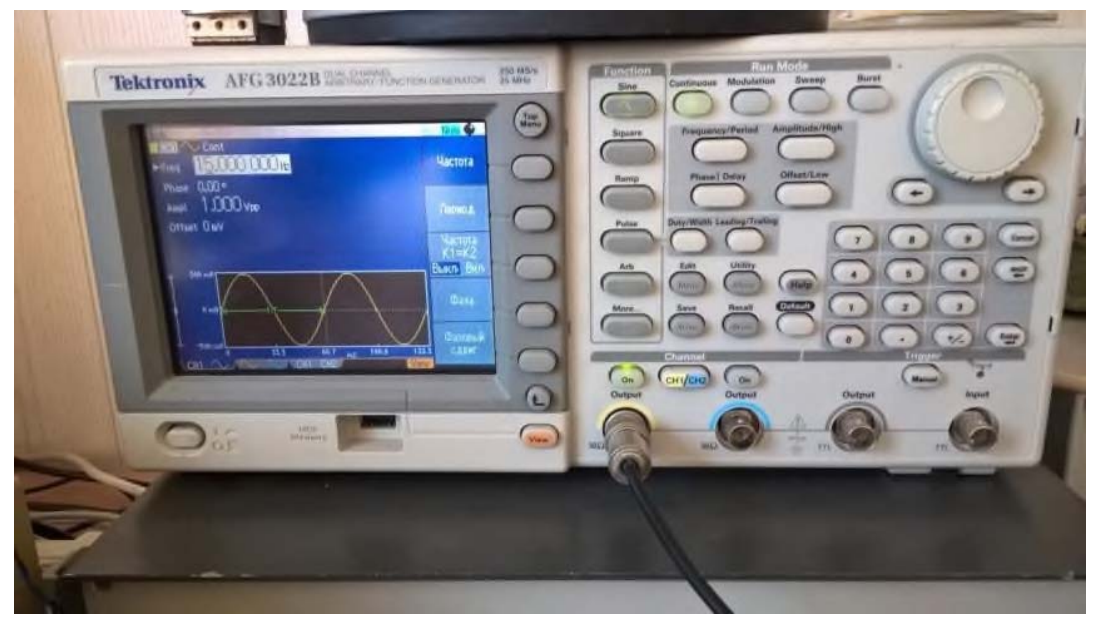

Fig. 2.13. Tektronix AFG 3022B Signal Generator

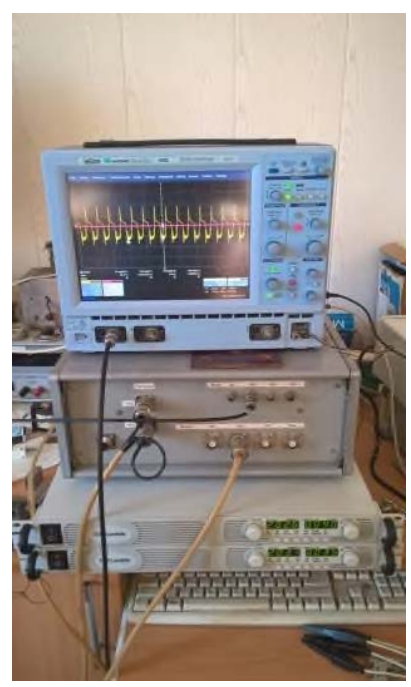

Fig. 2.14. Signal amplifier based on PA 94 piezo drivers (Apex Co., USA), TDK Lambda power supply and LeCroy 422 digital oscilloscope

Pictures 2.13 and 2.14 show photographs of the equipment used. Figure 2.15 shows a photograph of the cantilever-based setup. The conducted natural experiment showed that the first mode of vibrations of an ear with a stem (the first bending mode for the whole plant) is excited at a frequency of $2.6 \mathrm{~Hz}$, and the second mode in which the ear vibrates intensively with a relative rest of the stem was $15 \mathrm{~Hz}$, which is in good agreement with the calculations carried out earlier. 


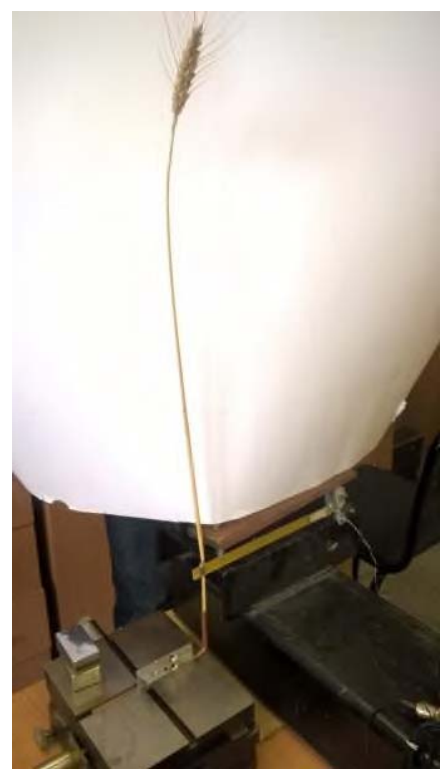

Fig. 2.15. Installation based on katilever

Figure 2.16 shows the second setup based on a round bimorph for high-frequency impact on the ear. The first three resonance frequencies obtained in the course of a fullscale experiment turned out to be $2.6 \mathrm{kHz}, 9.8 \mathrm{kHz}$, and $21.5 \mathrm{kHz}$, which is in good agreement with the calculations performed in Section 2.2. Figure 2.17 shows a photograph with a highlighted grain.

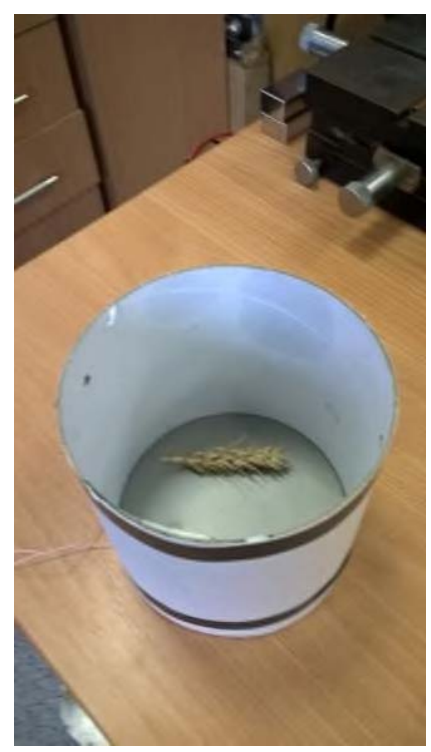

Fig. 2.16. Installation based on a round bimorph 


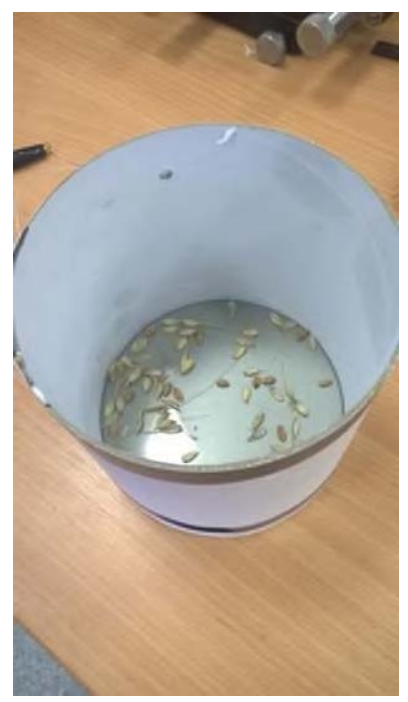

Fig. 2.16. Grain separation in a round bimorph-based plant

\section{Conclusion}

Mathematical and computer model in the ACELAN package of the resonance effect on the ear was developed and a field experiment was carried out, the results of which showed good agreement between the computer model of the present and previous research.

\section{References}

1. A. I. Buryanov, Improvement of the inertial method of harvesting grain crops by ochesom. Bulletin of Agrarian Science of the Don, 2(34), 15-23 (2016)

2. A. Altybayev, A. Zhanbyrbayev, B. Meskhi, D. Rudoy, A. Olshevskaya, A. Prohorova, E3S Web of Conferences, 135, 01078

(2019) https://doi.org/10.1051/e3sconf/201913501078

3. Y. Lachuga, A. Soloviev, A. Matrosov, I. Panfilov, V. Pakhomov, D. Rudoy, IOP Conf. Series: Earth and Environmental Science, 403, 012055IOP (2019) doi:10.1088/1755-1315/403/1/012055

4. E. N. Ziborov, A. A. Matrosov, Calculation of strength characteristics and service life of the front axle of a combine. State and prospects for the development of agricultural engineering: Proceedings of the 3rd international. scientific-practical Conf., Rostovon-Don, 199-200 (2010)

5. D. A. Kolesnikov, A. A. Matrosov, Calculation of strength characteristics and service life of the rear axle of the combine. State and prospects for the development of agricultural engineering: Materials of the 3rd international. scientific-practical Conf., Rostov-on-Don, 200-202 (2010)

6. A. A. Korotun, A. A. Matrosov, Calculation of strength characteristics and service life of the combine frame. State and prospects for the development of agricultural engineering: Proceedings of the 3rd international. scientific-practical Conf., Rostovon-Don, 217 (2010) 
7. O. I. Lysenok, A. A. Matrosov, Evaluation of the efficiency of the comb of the conveyor of the feeder chamber of the combine. State and prospects for the development of agricultural engineering: Proceedings of the 7th international. scientific-practical Conf., Rostov-on-Don, 86-87 (2014)

8. M. A. Buryanov, Methods of mathematical modeling of the interaction of plants of grain crops with the stripping drum of the header. Tractors and agricultural machines, 9, 19-22 (2015)

9. M. A. Buryanov, Methods of mathematical modeling of the process of grain movement in the transporting channel of the stripping header. Tractors and agricultural machines 10, 27-30 (2015)

10. M. A. Buryanov, Method of substantiating the parameters of the fairing of a singledrum stripping header. Tractors and agricultural machines, 7, 19-23 (2015)

11. B. Meskhi, B. Golev, V. Efros, D. Rudoy, A. Olshevskaya, V. Zhurba, Y. Chayka, E3S Web of Conferences, 135, $01083 \quad$ (2019) ITESE-2019 https://doi.org/10.1051/e3sconf/201913501083

12. Development of mathematical and computer models of dependencies and conditions for the separation of grain from an ear with various methods of physical and mechanical impact on it based on analytical, numerical methods and CAD-CAE complexes. REPORT under contract No. 0358100001618000013-0001658-01. 87, Rostov-on-Don (2018)

13. A. Zavaliy, S. Volozhaninov, O. Shiian1, D. Rudoy, A. Olshevskaya, E3S Web of Conferences, 175, $05003 \quad$ (2020) INTERAGROMASH 2020 https://doi.org/10.1051/e3sconf/202017505003

14. Yu. A. Ivanov, V. I. Pakhomov, S. I. Kambulov, D. V. Rudoi, (ICMTMTE 2018) electronic edition. Cep. MATEC Web of Conferences, 224, 05023 (2018) https://doi.org/10.1051/matecconf/201822405023

15. S. I. Bozhko, G. Parkhomenko, S. Kambulov, A. Boyko, V. Kolodkin, M. Magomedov, D. Rudoy, E3S Web of Conferences, 175, 05025 (2020) INTERAGROMASH $2020 \mathrm{https}$ ://doi.org/10.1051/e3sconf/202017505025

16. V. Zhurba, Y. Chayka, N. Gucheva, D. Ushakov, N. Ugrekhelidze, N. Kulikova, M. Egyan, E3S Web of Conferences, 135, 01087 (2019) ITESE-2019 https://doi.org/10.1051/e3sconf/201913501087 